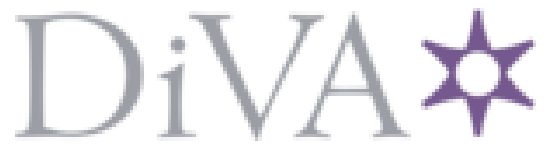

http://www.diva-portal.org

Postprint

This is the accepted version of a paper presented at Enterprise, Business-Process and Information Systems Modeling.

Citation for the original published paper:

Hersén, N., Hacks, S., Fögen, K. (2021)

Towards Measuring Test Coverage of Attack Simulations

In: (pp. 303-317). Springer International Publishing

https://doi.org/10.1007/978-3-030-79186-5_20

N.B. When citing this work, cite the original published paper.

Permanent link to this version:

http://urn.kb.se/resolve?urn=urn:nbn:se:kth:diva-297598 


\title{
Towards Measuring Test Coverage of Attack Simulations
}

\author{
Nicklas Hersén ${ }^{1}$, Simon Hacks $\square^{1}{ }^{[0000-0003-0478-9347]}$, and Konrad Fögen ${ }^{2}$ \\ 1 Division of Network and Systems Engineering \\ KTH Royal Institute of Technology, Stockholm, Sweden \\ \{nhersen|shacks\}@kth.se \\ 2 Research Group Software Construction \\ RWTH Aachen University, Aachen, Germany \\ foegen@swc.rwth-aachen.de
}

\begin{abstract}
Designing secure and reliable systems is a difficult task. Threat modeling is a process that supports the secure design of systems by easing the understanding of the system's complexity, as well as identifying and modeling potential threats. These threat models can serve as input for attack simulations, which are used to analyze the behavior of attackers within the system. To ensure the correct functionality of these attack simulations, automated tests are designed that check if an attacker can reach a certain point in the threat model. Currently, there is no way for developers to estimate the degree to which their tests cover the attack simulations and, thus, they cannot the determine the quality of their tests. To resolve this shortcoming, we analyze structural testing methods from the software engineering domain and transfer them to the threat modeling domain by following an Action Design Research approach. Further, we develop a first prototype, which is able to assess the test coverage in an automated way. This will enable threat modeler to determine the quality of their tests and, simultaneously, increase the quality of the threat models.
\end{abstract}

Keywords: Threat modeling $\cdot$ Attack simulations $\cdot$ Testing $\cdot$ Test coverage.

\section{Introduction}

Designing secure and reliable systems is a difficult task and attacker constantly find new ways to break into and compromise digital systems and infrastructures. Threat modeling is a process that involves understanding the complexity of a system, as well as identifying and modeling potential threats to the system or individual components [26]. The process involves modeling all potential weaknesses, threats, and vulnerabilities of a system. Often these are presented by means of graphs, where each of these is modeled as a single node and the transitions between them are expressed as edges [18]. Given a specific threat model of a system, attack simulations can be used to analyze specific attack scenarios on the described infrastructure $[4,9]$. The initial capabilities of an adversary are 
described as a set of initially compromised exploits or vulnerabilities, which in turn lead to other vulnerabilities the attacker can exploit in order to gain access to specific resources.

The meta attack language (MAL) [10] is a meta-language used to develop domain-specific languages (DSL) for threat modeling and defines which information about a system is required. Moreover, it specifies the generic attack logic. Then, a compiler can be used to generate a Java library from a MAL-based DSL, which can be used to programmatically create threat models. These models themselves are then used to perform attack simulations. More advanced simulations and analysis of threat models can be performed by using the generated jar file in applications such as securiCAD [4].

To ensure the quality of the created MAL-based DSLs, the language developers design tests that check the desired behavior of the language. Basically, they define a threat model and assess if the attack simulations on this model behave as expected. Currently, there is no way for developers to estimate how much of their created threat models are covered by existing tests. They can only manually assert whether specific attack steps are covered by the simulation or not. As the complexity of a threat model increases, it becomes harder to keep track of what parts have been tested resulting in many unnecessary overlapping tests. Furthermore, it is hard to determine how conclusive a test suite is without any type of coverage metric for a given threat model. Moreover, to the best of our knowledge, there exist no proposals how any test coverage for threat models could be measured nor is there a mapping from established coverage metrics.

Thus, we aim to develop a method to measure test coverage for threat models. In a first step, we develop a method to assess the coverage for a certain set of attack simulation to achieve this overall goal. Therefore, we consider classical coverage measurements from the software engineering domain and transfer them to attack simulations. Further, we implement an extension for the MAL that allows to automate the assessment of these test coverage.

The rest of the work is structured as follows. Next, we illustrate how we applied action design research to develop the coverage metrics presented in the section thereafter. This is followed by a introduction to the MAL and a description of the prototype to measure the coverage for attack simulations based on MAL. Finally, we give the related work before we conclude.

\section{Method}

In our research, we opt for an Action Design Research (ADR) [24] approach since we want to ensure a continuous feedback flow from the developer of MAL-based languages to the research team. ADR is characterized by four stages. Next, we illustrate how these stages are reflected in our research.

Stage 1: Problem Formulation Our research is driven by the need for a structured evaluation technique that helps to ensure the quality of MALbased languages. To the best of our knowledge, there exists no proposal for such measurement nor are the classical means of coverage assessment directly 
applicable, as the coverage is related to model instances and not a given source code. Therefore, we identify the common properties of attack graphs that are used for the attack simulations and research on common testing strategies within the software engineering domain. We opt for the software engineering domain because the identified properties of the attack graphs remind of logical flows in computer programs. Further, there exists a broad range of established tools for measuring coverage in this domain and we assume that we can easily adapt them to the properties of attack graphs.

Stage 2: Building, Intervention, and Evaluation In stage 2, we follow an IT-dominant approach. Using the terminology of ADR, one author act as researcher who is developing the test coverage metrics and a tool to measure them, while two authors provide their input as practitioners in the context of MAL-based language development and testing of software. Together, this team develops two kinds of artefacts: the coverage metrics and a tool to measure them. The end-users are presented by a set of MAL-based language developers who will use the tool in future to assess the coverage of their implemented test cases. Consequently, the coverage metrics are first evaluated with the authors representing the practitioners and, second, with the developers.

Stage 3: Reflection and Learning While in stage 2 a specific solution for a certain problem is developed, stage 3 focuses on the generalization of the solution so that it covers a class of problems. To achieve this generalization, we invert the proposed proceeding by developing first a general solution for measuring test coverage in attack graphs. Then, we implement a specific solution, which measures the test coverage in concrete attack graphs in relation to MAL.

Stage 4: Formalization of Learning The outcomes of stage 3 are formalized by a set of four different coverage metrics that can be reused in all contexts of attack simulation and their testing.

\section{Test Coverage for Attack Simulations}

In this section, we define first the fundamental properties of threat models and attack simulations. Then, we relate the concept of coverage metrics to these properties and, finally, discuss different metrics we developed for attack simulations.

\subsection{Fundamental Properties of Threat Models and Coverage}

To transfer the general concept of coverage to the domain of attack simulations, we need first to understand the fundamental properties of attack graphs and threat models, which serve as input for the attack simulations.

Threat modeling involves understanding the complexity of a system and identifying all possible security vulnerabilities regardless of whether they can be exploited [18]. This involves identifying sensitive parts of the system, such as possible attack entry point and sensitive resources. This process provides the developers with a high-level perspective of the system, which may be used to 
establish new security requirements, mitigate potential threats, or serve as a general guideline during the development process [21].

Attack graphs are a type of threat modeling used to describe vulnerabilities and potential exploits of a system. Formally, we understand an attack graph as a graph $G=(A, D, E, \mathfrak{A})$, with $A$ a set of nodes representing attack steps, $D$ a set of nodes representing defenses, $\mathfrak{A}$ a set of assets, and $E$ a set of edges linking either attack steps to each other or a defense to an attack step: $e \in E \subseteq\{(a, b)$ : $a \in A \cup D, b \in A\}$. Attack steps represent attacks, exploits, or vulnerabilities that can be leveraged in order to reach another system state [25]. An attack step $a \in A$ has a function $\tau: A \rightarrow\{$ AND, OR $\}$ denoted by $\tau(a)$. OR-type attack steps are only considered compromised if either of its parents are compromised, or if the step was initially compromised at the beginning of the simulation. In contrast, AND-type steps considered compromised if all parent attack steps have been compromised, or if its initial state was compromised.

A defense $d \in D$ has a function $\alpha: D \rightarrow\{$ TRUE, FALSE $\}$ denoted by $\alpha(d)$, which codifies if the attacker is hindered to assume related attack steps if TRUE $^{3}$. Lastly, the single nodes are grouped that they are related to certain assets that represent the fundamental components in the system under inspection [4]. These components can be any kind of hardware or software, depending on the desired abstraction level. Thus, we define an asset $\mathfrak{a}=\left(A^{\prime}, D^{\prime}, E^{\prime}\right) \mid \mathfrak{a} \in \mathfrak{A}, A^{\prime} \subseteq A, D^{\prime} \subseteq$ $D, E^{\prime} \subseteq E$ as a sub-graph representing a certain attack graph related to a cohesive component. All assets are distinct to each other, $\mathfrak{a}_{i} \bigcup \mathfrak{a}_{j}=\emptyset \mid \forall i \neq j$, so they share neither attack steps, defenses, nor edges. However, edges between the attack steps and defenses of two different assets are still possible that are not part of any asset, but of the overall attack graph $G$.

An attack simulation is a function $\phi:\left\{G, A_{s}, D_{a} \mid A_{s} \subseteq A, D_{a} \subseteq D\right\} \rightarrow$ $\left\{A_{c}, E_{c} \mid A_{c} \subseteq A, E_{c} \subseteq E\right\}$ denoted by $\phi(G, A, D)$. This function maps an attack graph, $G$, with a set of attack steps, $A_{s}$, that represent the attack surface of the attacker, and a set of defenses, $D_{a}$, that are in place, to a set of attack steps, $A_{c}$, and edges, $E_{c}$, that are compromised by the attacker.

With the fundamental properties of attack graphs and threat models being introduced, we now adapt the concept of coverage from the domain of software testing to provide information about what parts of the threat models are covered by an attack simulation.

Many structural software testing methods can be adapted to work with threat models and the generated simulation data. Common structural coverage criteria are node coverage, edge coverage and various types of path coverage [1]. In general, coverage is defined as the percentage of covered requirements or entities [17]:

$$
\text { Coverage }=100 \% \cdot \frac{\text { number of covered entities }}{\text { total number of entities }}
$$

\footnotetext{
${ }^{3}$ We are aware that defenses might be effective by a probability. However, testing non-deterministic behavior is challenging and, thus, we make the assumption that a defense is either effective or not.
} 
Software test data selection and generation is a big research topic and a lot of methods for producing valid and reliable test data have been proposed [5]. A poorly designed test suite may provide complete coverage of some software according to some model, but still fail to catch any errors. If code for dealing with edge cases is missing, structural coverage metrics will not account for these errors in the first place.

Similarly, the coverage results from attack simulations describe the coverage of the abstract threat model given some preconditions $\left(A_{s}, D_{a}\right)$. If the threat model does not adequately describe a specific system or infrastructure the results of attack simulations on that model might not correspond to the capabilities of an adversary attacking the actual system.

Testing the threat modeling could be considered as a system level test. The security researcher attempts to measure the capabilities of an attacker on an abstract model of a system or infrastructure. System level tests, and thus testing threat modeling, share a lot of similarities with code-based testing, the primary difference is where the structures representing different coverage metrics come from [20]. The coverage metrics essentially use common structure analysis techniques of the object under inspection, such as branch and node coverage with different coverage metrics [1].

\subsection{Exemplary Model}

Following, we illustrate an example that we use to demonstrate the calculation of the different coverage metrics for a specific threat model. The simulated scenario covers a situation where an adversary attempts to gain access to a target host $\mathrm{PC}$ protected by a password set by a specific user. The attack graph, $G^{e x}=$ $\left(A^{e x}, D^{e x}, E^{e x}, \mathfrak{A}^{e x}\right)$, contains three assets, $\mathfrak{A}^{e x}=\{$ Host, Password,User $\}$, with connected attack steps, $A^{e x}=\{$ authenticate, connect,$\ldots\}$, and a defense, $D^{e x}=$ \{encrypted $\}$, as shown in figure 1 . For the means of visualization, $\tau(a)=\mathrm{OR}$ is visualized using "|", $\tau(a)=$ AND using " \&", and a defense as "\#".

Initially the attacker is only able to connect to the target machine and is not able to influence the user in any way, only the connect and the authenticate step of the target machine are initially compromised $\phi\left(G^{e x},\{\right.$ connect, authenticate $\left.\}, D_{a}^{e x}\right)$. Two scenarios are simulated, one where the password is encrypted, $D_{a}^{e x}=$ $\{$ encrypted $\}$, and one where the password is not encrypted, $D_{a}^{e x}=\emptyset$.

In the following, we introduce new coverage metrics to calculate the coverage specific to attack steps, edges, assets, and defenses.

\subsection{Attack Step Coverage}

The simplest structural coverage is the node coverage which is often realized as statement coverage in source code testing [1]. A statement considers to be covered if there is a test case in which the statement is executed. Transferring this concept to attack simulations, a single attack step, $a \in A$, equals a node. Thus, we consider a certain attack step, $a$, as covered if there is an attack simulation, 
N. Hersén et al.

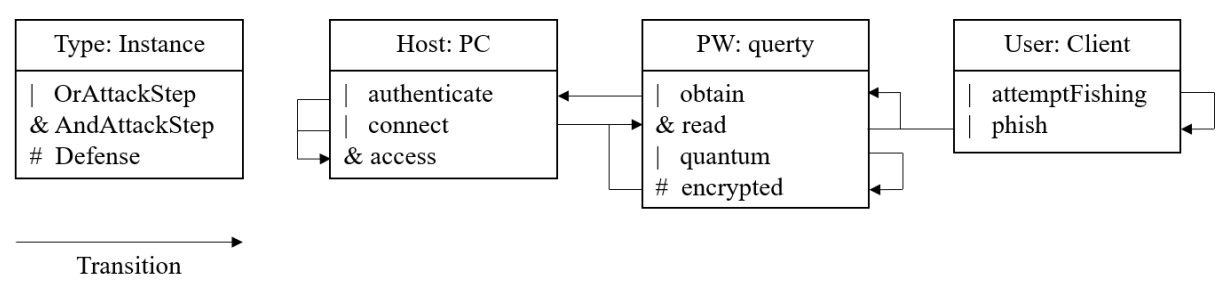

Fig. 1. Graphical representation of the threat model used for the example coverage calculations.

Table 1. Attack step coverage.

\begin{tabular}{l|cc|c} 
& $D_{a}^{e x}=\{$ encr. $\}$ & $D_{a}^{e x}=\emptyset \bigcup$ & \\
\hline$\left|A_{c}\right|$ & 3 & 5 & 5 \\
$|A|$ & 8 & 8 & 8 \\
\hline Cov. & $37.5 \%$ & $62.5 \%$ & $62.5 \%$
\end{tabular}

Table 2. Edge coverage.

\begin{tabular}{l|cc|c} 
& $D_{a}^{e x}=\{$ encr. $\}$ & $D_{a}^{e x}=\emptyset \bigcup$ & \\
\hline$\left|E_{c}\right|$ & 3 & 6 & 6 \\
$|E|$ & 9 & 9 & 9 \\
\hline Cov. & $33.3 \%$ & $66.7 \%$ & $66.7 \%$
\end{tabular}

$\phi(G, A, D)$, in which the attack step becomes compromised: $a \in A_{c}$. Then, let $A_{c}^{u}$ be the united set of all compromised attack steps along all attack simulations.

Consequently, the attack step coverage metric is computed as the ratio between the number of compromised attack steps, $\left|A_{c}^{u}\right|$, and the total number of attack steps, $|A|$. The attack step coverage percentage is computed as the ratio between them:

$$
P_{S}=\frac{\left|A_{c}^{u}\right|}{|A|}
$$

Table 1 illustrates an exemplary calculation of attack step coverage according to the two attack simulations described in Section 3.2. If the encryption is enabled, the attacker is able to additionally compromise access, but does not proceed further as read is protected. If the encryption is disabled, the attacker is able to compromise all attack steps of Host and two of Password but none of $U$ ser, as there is no edge from Password to User (just vice versa). Accordingly, the attack steps of $U$ ser are not compromised in any of our attack simulations.

\subsection{Edge Coverage}

After node coverage, authors usually define edge coverage as the next logical step that is most often implemented as branch coverage [1]. Here, we consider the edges between two attack steps, $E$, as equivalent to the edges in classical edge coverage. Accordingly, the edge coverage metric is computed as the ratio between all compromised edges $\left|E_{c}\right|$ and the number of edges $|E|$ in the graph:

$$
P_{E}=\frac{\left|E_{c}\right|}{|E|}
$$

Table 2 illustrates the calculation of edge coverage with respect to the two attack simulations described in Section 3.2. 
Table 3. Asset coverage.

\begin{tabular}{l|cc|c} 
& $D_{a}^{e x}=\{$ encr. $\}$ & $D_{a}^{e x}=\emptyset$ & $\bigcup$ \\
\hline $\mathfrak{A}_{\text {partial }}$ & 1 & 2 & 2 \\
$\mathfrak{A}_{\text {full }}$ & 1 & 1 & 1 \\
$|A|$ & 3 & 3 & 3 \\
\hline$P_{\text {partial }}$ & $33 \%$ & $67 \%$ & $67 \%$ \\
$P_{\text {full }}$ & $33 \%$ & $33 \%$ & $33 \%$
\end{tabular}

\subsection{Asset Coverage}

Next, we propose a metric for asset coverage, which is a metric for computing the percentage of compromised assets during an attack simulation. There exists no equivalently structural test coverage in the domain of software engineering [1]. The construct of class coverage for testing object-oriented software would be equivalent, but such a coverage would not provide any useful information. However, in attack simulations it is of relevance to know if an asset is covered. Unfortunately, there is not one solution to determine when an asset is interpreted as compromised since the requirements may vary depending on the situation. One approach would be to allow the developers to annotate the requirements to each asset. A simpler approach that we follow, is to use two separate definitions when we consider an asset to be compromised:

Full attack step coverage An asset, $\mathfrak{a}=\left(A^{\prime}, D^{\prime}, E^{\prime}\right)$, is considered to be compromised if all associated attack steps, $A^{\prime}$, have been compromised: $A^{\prime} \subseteq A_{c}^{u}$.

Partial attack step coverage An asset, $\mathfrak{a}=\left(A^{\prime}, D^{\prime}, E^{\prime}\right)$, is considered to be compromised if at least one associated attack step, $a^{\prime} \in A^{\prime}$, is compromised: $A^{\prime} \bigcup A_{c}^{u} \neq \emptyset$.

Let $\mathfrak{A}_{P}$ represent the set of all partially compromised assets, $C_{A}$ the set of all actually compromised assets and $\mathfrak{A}_{F}$ the set of fully compromised assets. The percentage of covered assets is computed as the ratio between one of the compromised asset sets and the set of all assets $\mathfrak{A}$ :

$$
P_{X}=\frac{\left|\mathfrak{A}_{X}\right|}{|\mathfrak{A}|} \mid X=\{\text { full, partial }\}
$$

Table 3 illustrates an exemplary calculation of partial and full asset coverage according to the model described in Section 3.2. In the first attack simulation, the Host gets fully compromised, while the attacker is not able to proceed to Password, due to the defense active. In the second attack simulation, succeeds to Password, but is not able to compromise it fully, due to quantum not reachable.

\subsection{Defense Coverage}

Defenses are a special because their value is set at the beginning of the simulation. Enabling and disabling defenses may result in different outcomes in multiple 


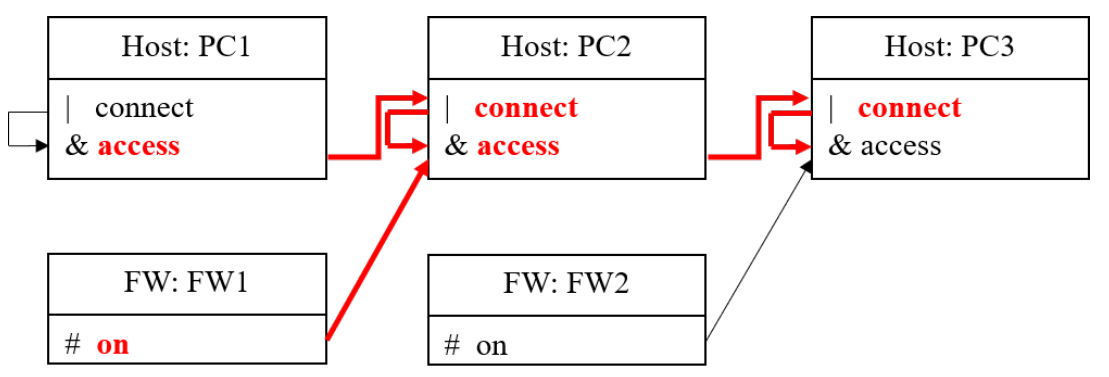

Fig. 2. Example of test covering all values for all defenses in a model. Red color represents that the attack step or edge is compromised.

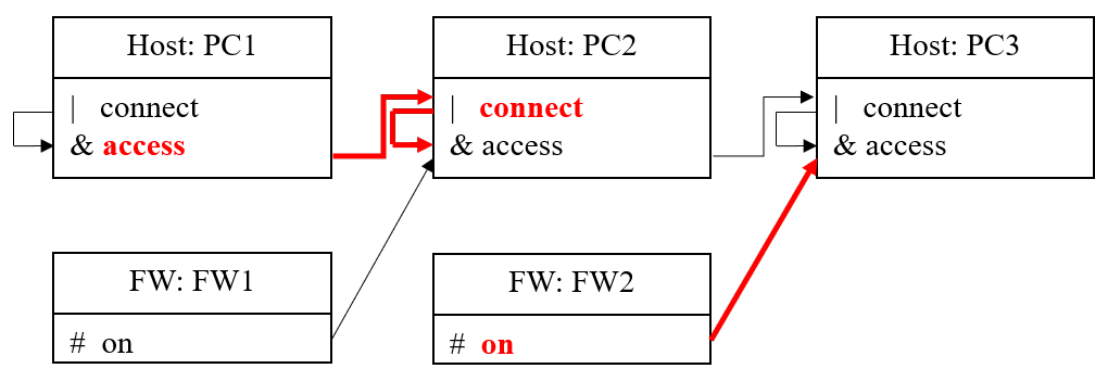

Fig. 3. Example of test covering all values for all defenses in a model. Red color represents that the attack step or edge is compromised.

executions of an attack simulation where the preconditions of the attacker do not change. Thus, each defense can be understood as an input parameter to the attack simulation and, consequently, interface-based input domain modeling [1] is the equivalent in the software engineering domain. For each input parameter a coverage criterion needs to be defined, usually by applying grouping the different inputs to classes. As the defenses are either in place or not, we consider just these two states. It may of interest for a security researcher to run a simulation with different defenses enabled to investigate how the opportunities of the attacker change according to the presence of certain defenses. Simply running simulations such that each defense has been enabled and disabled in at least one of the simulations may produce misleading results. Figure 2 and 3 presents two simulations such that all values of all defenses in the model have been covered.

From these results it may seem like the attacker is unable to compromise the access step of the rightmost host independent of the defenses state. In this model it is trivial to see that there exists a defense state such that the attacker is able to compromise the rightmost attack step.

Not running separate simulations for each defense state in the threat model under fixed preconditions may produce misleading results. This solution quickly becomes unfeasible since the growth to the defense state space is exponential 
relative to the number of defenses in the model $\left(2^{|D|}\right)$. Furthermore, running a simulation for different defense states only produces a coverage metric relevant for the current simulation.

For improved clarity, the notation of a simulation group is introduced which represents a set of simulations under some fixed precondition for different defense states $\left(D_{a}\right)$ :

$$
S G_{c o v}=\frac{\left|D_{a}\right|}{2^{|D|}}=\frac{2^{\log _{2}\left|D_{a}\right|}}{2^{|D|}}=2^{\log _{2}\left|D_{a}\right|-|D|}
$$

For models with multiple simulation groups the defense coverage metric is defined as the ratio between the sum of all simulated defense states in each simulation group, and the total number of defense states. Let $S=\left\{S_{1}, \ldots, S_{t}\right\}$ represent the set of all simulated simulation groups, where $S_{k}$ is the set of uniquely simulated defense states in simulation group $k$. The model defense coverage is described as follows:

$$
\frac{1}{t} \cdot \sum_{k=1}^{|S|} \frac{\left|S_{k}\right|}{2^{|D|}}=\frac{1}{2^{|D|} \cdot t} \cdot \sum_{k=1}^{t}\left|S_{k}\right|=\frac{1}{2^{|D|} \cdot t} \cdot 2^{\log _{2}\left(\sum_{k=1}^{t}\left|S_{k}\right|\right)}
$$

Then, the ratio of covered defense states for all simulation groups can be computed as follows:

$$
\operatorname{Cov}_{\mathcal{M}}=\frac{2^{\log _{2}\left(\sum_{k=1}^{t}\left|S_{k}\right|\right)-|D|}}{t}
$$

There is one defense in the example threat model presented in Section 3.2, resulting in a total of two defense states. Two simulations with the same initially compromised attack steps are executed with different defense configurations forming one simulation group. The resulting simulation group coverage is given by equation 5 , which results in the following defense simulation group coverage $(|D|=1,|C|=2)$ :

$$
S G_{C o v}=2^{\log _{2}|C|-|D|}=2^{0}=1
$$

The defense model coverage is given by equation 7 . Since both simulations in the example above are in the same simulation group, the model defense coverage metric is the same as the defense simulation group metric. The difference between the model and simulation group defense coverage metric is that the model version takes the different simulated groups into consideration when computing the coverage.

\section{Test Coverage for MAL-based Languages}

\subsection{The Meta Attack Language}

Next, we give a short presentation of the MAL. For a detailed overview of the MAL, we refer readers to the original paper [10]. First, a MAL-based DSL contains the main elements that are encountered on the domain under study, those 
are called assets in MAL. The assets contain attack steps, which represent the actual attacks/threats that can happen on them.

An attack step can be connected with one or more following attack steps so that an attack path is created. Those attack paths are then used to create attack graphs which are used for the attack simulation. Attack steps can be either of the type OR or of the type AND, respectively indicating that performing any individual parental attack step is required (OR) or all parental attack steps (AND) for the current step to be performed. Additionally, each attack step can be related with specific types of risks. Those risks can be any combination of confidentiality (C), integrity (I), and availability (A) and are specified in brackets after the attack step name. Furthermore, defenses are entities that do not allow connected attack steps to be performed if they have the value TRUE which represents them to be enabled. Finally, probability distributions can be assigned to the attack steps in order to represent the effort needed to complete the related attack step or the probability of the attack step to be possible.

Assets should also have relations between them in order for a model to be constructed, those relations are called associations in MAL. Inheritance between assets is also possible and each child asset inherits all the attack steps of the parent asset. Additionally, the assets can be organized into categories for purely organization reasons.

In Listing 1, a short example of a MAL-based DSL is presented. In this example, four modeled assets can be seen together with the connections of attack steps from one asset to another. In the Host asset, the connect attack step is an OR attack step while access is an AND attack step. Then, the - $>$ symbol denotes the connected next attack step. For example, if an attacker performs phish on the User, it is possible to reach obtain on the associated Password and as a result finally perform authenticate on the associated Host. In the last lines of the example the associations between the assets are defined.

\subsection{Implementation of a Prototype}

Hitherto, we have developed four different test coverage metrics for attack simulations and introduced MAL on which we want to measure these coverages. Thus, we gather the coverage data by an extension to JUnit 5 on a system-level for specific threat models, which is available on GitHub ${ }^{4}$. Data gathered by the extension is a measurement of the capabilities of a simulated attacker on a specific model. The extension of JUnit was necessary, since the existing coverage measurements do not map one to on to the proposed measurements for attack graphs. This is caused by the fact that the attack graphs are translated into different classes. Thus, a statement coverage of these classes does not express anything e.g., on the attack step coverage, because attack steps are expressed as values in a list.

Since the extension does not have access to the original MAL language specification there is no metric of how much of the DSL is covered by existing tests.

\footnotetext{
${ }^{4}$ https://github.com/nicklashersen/malcompiler
} 
The coverage extension supports custom exportable targets by extending the ExportableTarget class. Exportable targets are responsible for computing and formatting coverage data generated by each JUnit test. The ExportableTarget class does not provide any functions for computing coverage metrics. As a result, there is no common coverage metric included by default for each deriving class. Methods for accessing attack step and defense objects associated with a specific asset are provided, but the responsibility for deciding what information to include is delegated to the deriving classes.

The ConsoleTarget is an extension of the ExportableTarget class based on the coverage metrics discussed in section 3. Coverage data is collected after each attack simulation and stored for further processing during the export phase. A ModelKey is computed for each model used in the simulations associating test-methods with specific threat-models. Each test-method gets assigned to a simulation group based on the initially compromised attack steps. Since each test method can only be in one defense state, the defense coverage metric is only printed for simulation groups and model coverage metrics.

All coverage data is processed and presented when the export method is called. The coverage metrics for simulation groups and models is represents the compounded result of all child simulations. If an asset, attack step or edge is compromised in at least one child simulation, the object will be considered compromised in the parent simulation group or model metric.

Alternatively, the results can be exported to a JSON file. To ease the understanding of the output, we have developed a dedicated viewer for these JSON files. The viewer is available on Github ${ }^{5}$.

\section{Related Work}

Next, we provide an overview of previously conducted research related to the subject of study. First, we elaborate on model-driven security, which stems from research on extending the UML language for secure systems development. One of the earliest extensions of UML with focus on secure system development was proposed by Jürjens [11]. His proposal focused on extending the semantics of existing UML types for better expressing and integrating security related information such as security requirements, concepts, and threats for components in a UML diagram [11]. Another security focused UML extension was presented by Lodderstedt et. al called secureUML. Rather than providing general threat modeling functionality similar to UMLsec the secureUML extension provides tools for modeling role-based access control and authorization constraints.

Another common threat modeling process is to represent potential exploits and vulnerabilities of a system as an attack graph. The concept of attack graphs is generally considered to be introduced by Schneier in the form of attacktrees [23] and later formalized by Sjouke \& Martijn [16]. A limitation with attack trees is that they fail to capture the interaction between attacks and defensive

\footnotetext{
${ }^{5}$ https://github.com/nicklashersen/mal-coverage-viewer
} 
measures present in the modeled infrastructure. Kordy et al. expanded the concept of attack trees by introducing defenses (attack-defense trees) to better capture the evolution of security mechanisms and vulnerabilities in a system [14].

Using simulations to provide insight over system operation and for evaluating system requirements is a common practice in many fields, like network security. Cohen [3] suggested a network security model consisting of a network model, a cause-effect model, characteristic function, and pseudo-random number generators for simulating attacks, defenses, and consequences. However due to the simplicity of the cyber-attacks or defense representations based on the causeeffect model, any practical application of the model may prove difficult [2].

Quantifying coverage of potential attacks and threats for a specific model is a difficult task. Domain experts are often required to make a judgment based on prior experience on what type of tests are necessary to provide adequate coverage over relevant attacks and threats for specific systems. Many threat modeling tools do not help with the task of determining when sufficient coverage has been reached, but rather focus on providing estimates of an attacker's capabilities through different metrics. For example, SecureITree [15] models a system through the use of attack-trees and estimates the risk of a specific attack occurring as the product between the incident probability and impact. There are other techniques that work in a similar manner but are based on other metrics, such as the attack and damage cost [22]. These metrics do not provide an estimate on whether sufficiently many attackers have been simulated, but they may provide insight over potential vulnerabilities of the models.

Automated security testing tools tend to provide more easily quantifiable coverage metrics. In a proposal for testing of security policies for firewalls, the security policies were represented as directed acyclic graphs (DAGs). Test cases represented each complete event sequence which could be derived from the DAG [27]. System testing and code-based coverage analysis employ similar testing methodologies. The primary difference is where components used to describe the system (graphs, clauses, etc.) come from [20].

The Meta Attack Language (MAL) is a language for creating domain specific cyber threat modeling systems and attack simulations [10]. While there has been a lot of research on the field of threat modeling, there seems to be little research related to threat modeling and attack simulations in the field of domain specific languages (DSL) [19]. There have been attempts to extend the cause-effect model by employing advanced modeling and simulation concepts for specific domains to support threat modeling and attack simulations [2].

While the MAL is a relatively new language there already exist a number of MAL based DSLs for different domains, like vehicleLang [13], which allows modeling cyber-attacks on modern vehicles. Other languages are coreLang [12], which contains the most common IT entities and attack steps, or powerLang [8] that is designed for modeling organizations in the power domain. Another approach is the automated creation of MAL languages by translating existing concepts from the ArchiMate language to MAL [7]. 


\section{Conclusion}

The goal of this work was to develop a method to measure test coverage for attack simulations and to provide a prototype that allows the automated measurement for MAL-based languages. We proposed four coverage metrics based on existing coverage criteria and implemented them. These metrics provide different estimates on how much and what parts of a threat model are covered by a set of tests. This is valuable feedback for the language developers because it supports the decision if further testing is needed.

Since our work is to the best of our knowledge, a new approach to secure the quality of threat models and attack simulations, there are a lot of potential improvements and new features that could be added. Firstly, there exist many different and more advanced coverage metrics like path coverage, modified condition/decision coverage (MC/DC), or active clause coverage (ACC) [1], which could serve as valuable coverage metrics. Secondly, a common metric interface could be designed to separate the coverage metric code from the exportable targets. This could be used to facilitate the reuse of existing coverage metrics during the development of new coverage metrics. Further, we consider defenses either as active or not, while in reality defenses are probably effective. Thus, further research is needed to consider this in our framework, too.

A shortcoming of our work is that the prototype only computes the coverage of assets used in the attack simulations. If an asset defined in the language is never used, the asset will not be included in the coverage computation.

\section{Acknowledgement}

This project has received funding from the European Union's H2020 research and innovation programme under the Grant Agreement No. 832907.

\section{References}

1. Ammann, P., Offutt, J.: Introduction to Software Testing. Cambridge University Press, New York, NY, USA, 2nd edn. (2016)

2. Chi, S.D., Park, J.S., Jung, K.C., Lee, J.S.: Network security modeling and cyber attack simulation methodology. In: Australasian Conference on Information Security and Privacy. pp. 320-333. Springer (2001)

3. Cohen, F.: Simulating cyber attacks, defences and consequences. Computers \& Security 18(6), 479-518 (1999)

4. Ekstedt, M., Johnson, P., Lagerström, R., Gorton, D., Nydrén, J., Shahzad, K.: securiCAD by foreseeti: A CAD tool for enterprise cyber security management. In: 19th International EDOC Workshop. pp. 152-155. IEEE (2015)

5. Goodenough, J.B., Gerhart, S.L.: Toward a theory of test data selection. IEEE Transactions on software Engineering (2), 156-173 (1975)

6. Gulati, S., Sharma, R.: JUnit 5 Extension Model, pp. 121-137. Apress, Berkeley, CA (2017) 
7. Hacks, S., Hacks, A., Katsikeas, S., Klaer, B., Lagerström, R.: Creating meta attack language instances using archimate: Applied to electric power and energy system cases. In: 23rd International EDOC. pp. 88-97 (2019)

8. Hacks, S., Katsikeas, S., Ling, E., Lagerström, R., Ekstedt, M.: powerlang: a probabilistic attack simulation language for the power domain. Energy Inform 3(1) (2020)

9. Holm, H., Shahzad, K., Buschle, M., Ekstedt, M.: P²CySeMoL: Predictive, probabilistic cyber security modeling language. IEEE Trans Dependable Secure Comput 12(6), 626-639 (2015)

10. Johnson, P., Lagerström, R., Ekstedt, M.: A meta language for threat modeling and attack simulations. In: 13th ARES Conference. pp. 1-8 (2018)

11. Jürjens, J.: Umlsec: Extending uml for secure systems development. In: International Conference on The Unified Modeling Language. pp. 412-425. Springer (2002)

12. Katsikeas, S., Hacks, S., Johnson, P., Ekstedt, M., Lagerström, R., Jacobsson, J., Wällstedt, M., Eliasson, P.: An attack simulation language for the it domain. In: Graphical Models for Security. pp. 67-86. Springer, Cham (2020)

13. Katsikeas, S., Johnson, P., Hacks, S., Lagerström, R.: Probabilistic modeling and simulation of vehicular cyber attacks : An application of the meta attack language. In: 5th ICISSP (2019)

14. Kordy, B., Mauw, S., Radomirović, S., Schweitzer, P.: Foundations of attackdefense trees. In: Degano, P., Etalle, S., Guttman, J. (eds.) Formal Aspects of Security and Trust. pp. 80-95. Springer Berlin Heidelberg, Berlin, Heidelberg (2011)

15. Limited, A.T.: Hostile risk decisions and capabilities-based analysis (2020), https: //www.amenaza.com/downloads/docs/Hostile\%20Risk\%20Decisions.pdf

16. Mauw, S., Oostdijk, M.: Foundations of attack trees. In: International Conference on Information Security and Cryptology. pp. 186-198. Springer (2005)

17. Miranda, B., Bertolino, A.: Testing relative to usage scope: Revisiting software coverage criteria. ACM Trans. Softw. Eng. Methodol. 29(3) (Jun 2020)

18. Myagmar, S., Lee, A.J., Yurcik, W.: Threat modeling as a basis for security requirements. In: SREIS. vol. 2005, pp. 1-8. Citeseer (2005)

19. do Nascimento, L.M., Viana, D.L., Neto, P., Martins, D., Garcia, V.C., Meira, S.: A systematic mapping study on domain-specific languages. In: ICSEA 2012. pp. 179-187 (2012)

20. Offutt, J., Liu, S., Abdurazik, A., Ammann, P.: Generating test data from statebased specifications. Software testing, verification and reliability 13(1), 25-53 (2003)

21. Oladimeji, E.A., Supakkul, S., Chung, L.: Security threat modeling and analysis: A goal-oriented approach. In: SEA 2006. pp. 13-15. Citeseer (2006)

22. Saini, V., Duan, Q., Paruchuri, V.: Threat modeling using attack trees. Journal of Computing Sciences in Colleges 23(4), 124-131 (2008)

23. Schneier, B.: Attack trees. Dr. Dobb's journal 24(12), 21-29 (1999)

24. Sein, M.K., Henfridsson, O., Purao, S., Rossi, M., Lindgren, R.: Action design research. MIS Quarterly 35(1), 37-56 (2011)

25. Sheyner, O., Haines, J., Jha, S., Lippmann, R., Wing, J.M.: Automated generation and analysis of attack graphs. In: Proceedings 2002 IEEE Symposium on Security and Privacy. pp. 273-284. IEEE (2002)

26. Shostack, A.: Threat modeling: Designing for security. Wiley, Indianapolis, IN, USA (2014)

27. Tuglular, T., Kaya, Ö., Müftüoglu, C.A., Belli, F.: Directed acyclic graph modeling of security policies for firewall testing. In: International Conference on Secure Software Integration and Reliability Improvement. pp. 393-398. IEEE (2009) 